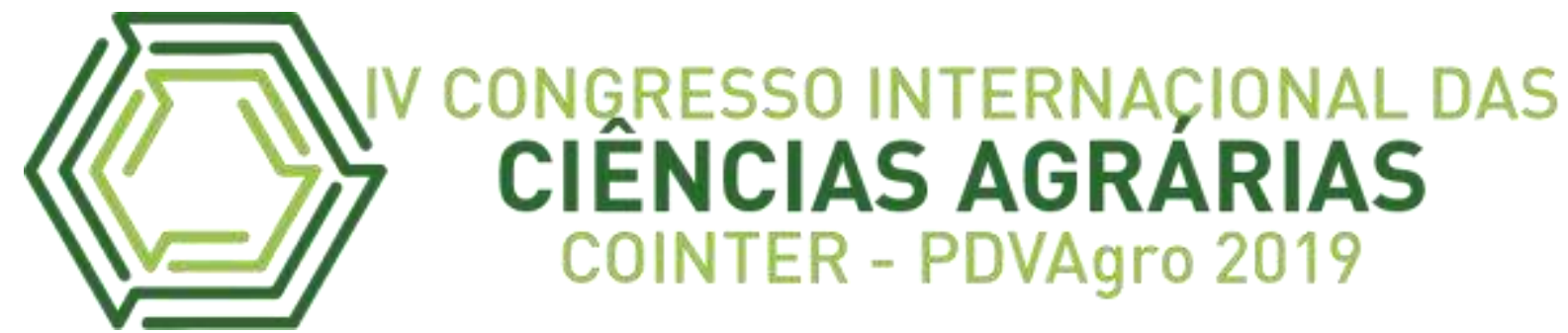

\title{
ATRIBUTOS BIOMÉTRICOS DE FRUTOS E SEMENTES DE Morinda citrifolia (L.) PROVENIENTES DO MUNICÍPIO DE BOQUIRA - BA
}

\section{ATRIBUTOS BIOMÉTRICOS DE FRUTOS Y SEMILLAS DE Morinda citrifolia $(\mathrm{L}$. DE BOQUIRA - BA}

\section{BIOMETRIC ATTRIBUTES OF FRUIT AND SEEDS OF Morinda citrifolia (L.) FROM BOQUIRA - BA}

\author{
Jamil Sousa Silva ${ }^{1}$; Emanuelle Marques Coutinho²; Mateus Pereira dos Santos ${ }^{3}$; Zenóbia \\ Cardoso dos Santos ${ }^{4}$; Gisele Brito Rodrigues ${ }^{5}$ \\ Apresentação: Comunicação Oral
}

DOI: https://doi.org/10.31692/2526-7701.IVCOINTERPDVAgro.2019.0074

\begin{abstract}
Resumo
O noni (Morinda citrifolia Linn) é uma espécie exótica que tem sido utilizada no tratamento de doenças. No Brasil, seu cultivo tem crescido nos últimos anos, principalmente nas regiões semiáridas do Nordeste, porém, poucos estudos relacionados às características morfológicas de seus frutos e sementes têm sido desenvolvidos. Assim, objetivou-se com este trabalho, caracterizar os frutos e sementes de noni provenientes do município de Boquira, BA. O estudo foi conduzido no Laboratório de Tecnologia e Produção de Sementes da Universidade Estadual do Sudoeste da Bahia - UESB. Para o estudo de biometria foram coletados frutos de diferentes matrizes do município de Boquira - BA, selecionados e separados 100 unidades sem sinal de ataque de patógenos ou insetos. As sementes foram extraídas dos frutos manualmente por meio de uma peneira. As características analisadas para os frutos foram: comprimento $(\mathrm{mm})$, diâmetro $(\mathrm{mm})$ e massa fresca $(\mathrm{g})$, e para as sementes, avaliou-se o comprimento $(\mathrm{mm})$, largura (mm) e espessura $(\mathrm{mm})$. Os dados obtidos foram submetidos à análise estatística descritiva, distribuição de frequências e cálculos de coeficiente de correlação de Spearman (rS). Os frutos e sementes de noni apresentaram variabilidade para todas as características avaliadas. Foram encontradas correlações positivas e significativas entre comprimento e diâmetro de frutos, enquanto para sementes, verificou-se correlação significativa positiva para o comprimento e largura, e comprimento e espessura das sementes. Recomenda-se a escolha de matrizes com

\footnotetext{
${ }^{1}$ Curso de Agronomia, Universidade Estadual do Sudoeste da Bahia - UESB, e-mail: jamilsousa13@gmail.com

2 Curso de Agronomia, Universidade Estadual do Sudoeste da Bahia - UESB, e-mail: emanuellemarques321@gmail.com

3 Graduado em Agronomia, Universidade Estadual do Sudoeste da Bahia - UESB, e-mail: mateus.santos.0712@gmail.com

${ }^{4}$ Programa de Pós-Graduação em Agronomia (Fitotecnia), Universidade Estadual do Sudoeste da Bahia - UESB, e-mail: cardosoz@yahoo.com

5 Docente Doutora do curso de Agronomia - Universidade Estadual do Sudoeste da Bahia (UESB), gisele.rodrigues@uesb.edu.br
} 
base no comprimento de frutos e de sementes.

Palavras-Chave: biometria, Rubiaceae, espécie exótica, noni

\title{
Resumen
}

El Noni (Morinda citrifolia Linn) es una especie exótica que se ha utilizado para tratar enfermedades. En Brasil, su cultivo ha crecido en los últimos años, principalmente en las regiones semiáridas del noreste, sin embargo, se han desarrollado pocos estudios relacionados con las características morfológicas de sus frutos y semillas. Por lo tanto, el objetivo de este trabajo fue caracterizar las frutas y semillas de noni de Boquira, BA. El estudio se realizó en el Laboratorio de Tecnología y Producción de Semillas de la Universidad Estatal del Suroeste de Bahía - UESB. Para el estudio biométrico se recolectaron frutos de diferentes matrices de Boquira - BA, seleccionadas y separadas 100 unidades sin signos de ataque de patógenos o insectos. Las semillas se extrajeron de las frutas a mano a través de un tamiz. Las características analizadas para los frutos fueron: longitud $(\mathrm{mm})$, diámetro $(\mathrm{mm})$ y masa fresca $(\mathrm{g})$, y para las semillas, se evaluaron la longitud $(\mathrm{mm})$, el ancho $(\mathrm{mm})$ y el grosor $(\mathrm{mm})$. Los datos obtenidos se sometieron a análisis estadísticos descriptivos, distribución de frecuencias y cálculos del coeficiente de correlación de Spearman $(\mathrm{rS})$. Las frutas y semillas de noni mostraron variabilidad para todas las características evaluadas. Se encontraron correlaciones positivas y significativas entre la longitud y el diámetro de las frutas, mientras que para las semillas hubo una correlación positiva significativa para la longitud y el ancho, y la longitud y el grosor de las semillas. Se recomienda elegir matrices basadas en la fruta y la longitud de la semilla.

Palabras Clave: biometría, Rubiaceae, especies exóticas, noni.

\begin{abstract}
Noni (Morinda citrifolia Linn) is an exotic species that has been used to treat diseases. In Brazil, its cultivation has grown in the last years, mainly in the semiarid regions of the Northeast. However, few studies related to the morphological characteristics of its fruits and seeds have been developed. Thus, the objective of this work was to characterize noni fruits and seeds from Boquira, BA. The study was conducted at the Laboratory of Technology and Seed Production of the State University of Southwest Bahia - UESB. For the biometrics study were collected fruits from different matrices of Boquira - BA, selected and separated 100 units without signs of attack of pathogens or insects. The seeds were extracted from the fruits by hand through a sieve. The characteristics analyzed for the fruits were: length $(\mathrm{mm})$, diameter $(\mathrm{mm})$ and fresh mass $(\mathrm{g})$, and for the seeds, the length $(\mathrm{mm})$, width $(\mathrm{mm})$ and thickness $(\mathrm{mm})$ were evaluated. The data obtained were submitted to descriptive statistical analysis, frequency distribution and Spearman correlation coefficient (rS) calculations. Noni fruits and seeds showed variability for all evaluated characteristics. Positive and significant correlations were found between length and diameter of fruits, while for seeds there was a significant positive correlation for length and width, and length and thickness of seeds. It is recommended to choose matrices based on fruit and seed length.
\end{abstract}

Keywords: biometry, Rubiaceae, exotic species, noni.

\section{Introdução}

A espécie Morinda citrifolia (L.), conhecida popularmente no Brasil como Noni, 
apresenta potencialidades para uso medicinal, sendo utilizada no tratamento de saúde devido às suas propriedades antioxidantes, além de ser apreciado por algumas pessoas, que consomem o fruto na forma in natura ou processada (SOARES et al., 2013; NASCIMENTO et al., 2016).

É uma espécie exótica, pertencente à família Rubiaceae, tendo como centro de origem a região Sudeste do Continente Asiático, que está adaptada a condições edafoclimáticas diversas, podendo se desenvolver em florestas tropicais e em regiões de solos rochosos ou arenosos. Apresenta grande distribuição geográfica, ocorrendo em várias regiões da África, China, Indonésia, Caribe, Índia Austrália entre outras.

No Brasil, as plantas exóticas são objetos de poucos estudos quanto aos seus benefícios, como a procura por diferentes fontes alimentares e síntese de novos princípios ativos de utilidade farmacêutica entre outras diversas aplicações (COSTA et al., 2013; PALIOTO et al., 2015; LIMA et al., 2018). Esse é o caso do noni, que apesar de possuir características fitoterápicas importantes, é pouco utilizado no país, porém, acredita-se que, seu cultivo pode vir a apresentar importância econômica, principalmente por se tratar de uma planta adaptada às condições tropicais e por poder contribuir com a descoberta de novos princípios ativos com fins farmacêuticos de seus frutos (MATOSO et al., 2013).

Sabe-se que, o crescimento e desenvolvimento vegetal sofrem influência de fatores abióticos como temperatura, radiação solar e precipitação (BERILLI et al., 2007), e esses fatores, juntamente com as características genéticas da espécie, podem exercer efeitos na reprodução e desenvolvimento dos frutos e sementes.

Segundo Moura et al. (2003) os frutos passam por uma série de processos fisiológicos durante o seu desenvolvimento, resultando em mudanças na aparência externa, tamanho, forma, cor e textura de suas estruturas e tecidos, sabor, odor e valor nutritivo na formação final do fruto. Essas mudanças possibilitam a determinação do ponto colheita dos frutos e sementes, em sua fase de máxima qualidade (CARVALHO e NAKAGAWA, 2012).

Dessa forma, determinar os atributos biométricos dos frutos podem ser ferramentas úteis para auxiliar os produtores quanto ao momento mais adequado para executar a colheita dos frutos no seu estado de maior qualidade, além de ter importância ecológica, como a detecção de variabilidade genética entre as populações de uma determinada espécie.

Todavia, não existem relatos sobre a características biométricas de frutos e sementes de noni, sendo necessários desenvolver estudos que forneçam informações sobre a ecologia dessa espécie e sua interação com o ambiente, que possibilitem a aplicação em sistemas de 
produção afim de incrementar a produtividade (CANUTO et al., 2010; NEVES, 2012).

Nesse sentido, o objetivo desse trabalho foi avaliar os aspectos biométricos de frutos e de sementes de Morinda citrifolia (L.) provenientes do município de Boquira, Bahia.

\section{Fundamentação Teórica}

O Brasil vem ganhado posição de destaque, na produção e comercialização de frutas nativas e exóticas, motivada pela crescente busca do consumidor, por produtos benéficos a saúde, os denominados alimentos funcionais (SINGH, 2012).

Muitas plantas nativas e exóticas com propriedades medicinais têm sido utilizadas no tratamento de enfermidades, também conhecida como fitoterapia, sendo considerada uma das práticas mais antigas da humanidade. A fitoterapia tem o objetivo de extrair princípios ativos produzidos pelas plantas que sejam capazes de curar ou prevenir doenças e ferimentos. No Brasil, a fitoterapia tem sido historicamente praticada pela população indígena, se consolidando até os dias de hoje como um segmento da medicina alternativa e naturalista. Os nativos detinham certo conhecimento sobre o poder curativo de algumas espécies botânicas que promoviam ou acelerava a cura de determinadas enfermidades (BRASIL, 2015).

Atualmente, frutos de plantas exóticas como o Noni (Morinda citrifolia L.), são utilizadas pela população por apresentar características fitoterápicas, como ações antiinflamatória, antimicrobiana, analgésica, hipotensora e anticarcinogênica (BARBOSA et al., 2017).

A espécie Morinda citrifolia é uma planta exótica que pertence à Família Rubiaceae (Subfamília Rubioideae) e foi introduzida no Brasil por meio de sementes trazidas da região do Caribe e da Polinésia. Esta espécie apresenta grande porte podendo atingir até 10 metros de altura, suas folhas são largas e simples, de coloração verde escura, a produção de flores e frutos ocorre durante todo o ano, sendo que as flores são pequenas e brancas, enquanto que os frutos são ovoides, de coloração branca meio esverdeada.

Os frutos de noni são constituído por $90 \%$ de água, sendo que os principais componentes da matéria seca são sólidos solúveis, fibras, proteínas e carboidratos. O peso médio está em torno de 205 gramas, além disso, as dimensões podem ser de 12 centímetros de comprimento e $6 \mathrm{~cm}$ de largura, podendo conter entre 240 a 280 sementes. Nos primeiros estágios de desenvolvimento, os frutos apresentam a coloração verde e à medida que vai se desenvolvendo, 
a coloração passa a ser amarela e quando maduros, apresentam a cor branca, indicando o momento adequado para a colheita (BECHELENI, 2017).

A adaptação de $M$. citrifolia ao clima tropical, possibilitou o seu cultivo no Brasil, onde, atualmente é realizado em pequenos pomares, em sua grande maioria na região Nordeste. No entanto, o uso do noni para fins medicinais tem sido realizado de forma inadequada quanto a sua real função fitoterápica. Além disso, trabalhos relacionados à eficácia e propriedades terapêutica das diversas parte da planta estão sendo desenvolvidos e os resultados tem indicado informações controvérsias (BARBOSA et al., 2017).

De acordo com estudos, o consumo do noni no Brasil, seja como alimento ou como medicamento tem aumentado significativamente nos últimos anos, sendo que o fruto in natura pode ser adquirido no comércio através de venda direta e os medicamentos em farmácias de manipulação. Todavia, há controvérsias quanto ao uso do noni por parte da população, sendo que no ano de 2007, a Agência Nacional de Vigilância Sanitária (ANVISA) divulgou uma nota que advertia que produtos à base de noni não deveriam ser comercializados no Brasil como alimento, até que seja comprovada a sua segurança de uso (FARIA et al. 2014).

Existem poucos estudos na literatura científica que fazem referência às características biométricas dos frutos e sementes da espécie $M$. citrifolia, dessa forma, as características agronômicas da planta acabam sendo pouco exploradas (OLIVEIRA et al. 2011).

A biometria de frutos e sementes fornece informações para a conservação e exploração dos recursos de valor econômico, possibilitando o uso adequado e racional dos frutos, bem como pode ser utilizada na avaliação da variabilidade genética inter e intrapopulacional e da relação, ou não, desta variabilidade com os fatores edafoclimáticos. Além disso, possibilita traçar uma estratégia para maximizar a uniformização de emergência das plântulas e obter mudas de padrões semelhantes ou de maior vigor através da classificação das sementes por tamanho e por peso. A biometria dos frutos e sementes também é relevante para diferenciar a intensidade de variação das espécies relacionada a fatores ambientais, como as reações das populações quando estão estabelecidas em outro ambiente (CARVALHO; NAKAGAWA, 2012; BARROSO et al., 2016).

Diante da biodiversidade frutífera que o Brasil apresenta e seus benefícios faz se necessário, maior estudo destes frutos ainda pouco caracterizados e explorados por pesquisadores. Estudos aprofundados podem ajudar no processo de desenvolvimento de novos produtos para melhoria na alimentação e saúde da população. 


\section{Metodologia}

O estudo foi conduzido no Laboratório de Tecnologia e Produção de Sementes da Universidade Estadual do Sudoeste da Bahia - UESB, Campus de Vitória da Conquista -BA.

Os frutos foram coletados em janeiro de 2019, diretamente da copa de sete árvores matrizes quando apresentavam coloração amarela, que corresponde ao estágio de maturidade dos frutos. As matrizes estão localizadas em Boquira-BA, no município inserido na região da Chapada Diamantina, onde o clima varia entre semiárido à subúmido a seco, com temperatura média anual de $23,8^{\circ} \mathrm{C}$ e pluviosidade anual de $894,8 \mathrm{~mm}$ (SEI, 2011).

Após a colheita, os frutos foram acondicionados em sacos plásticos e encaminhadas ao laboratório para o estudo da biometria. Em laboratório, foram selecionados aleatoriamente 100 frutos sem sinais de ataque de patógenos e sem deformações. As análises biométricas (Figura 1) foram executadas com o auxílio de um paquímetro digital de precisão de $0,05 \mathrm{~mm}$, sendo determinados:

- Comprimento longitudinal: determinado da base até o ápice do fruto;

- Diâmetro: medido na linha mediana central de cada fruto;

- Massa fresca do fruto: determinada através de pesagem de cada fruto em balança analítica com precisão de $0,001 \mathrm{~g}$.

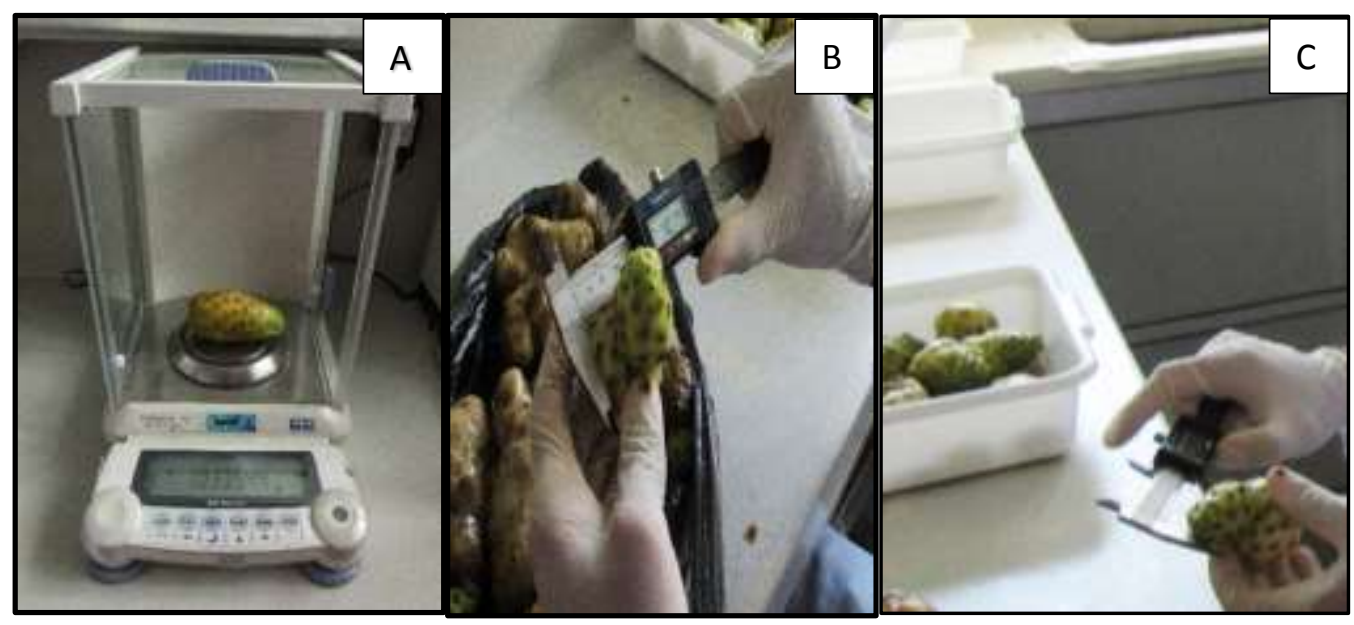

Figura 1. Massa fresca do fruto (A), Diâmetro (B) e Comprimento longitudinal (C)

Para a avaliação das características biométricas das sementes, procedeu-se o beneficiamento dos frutos, removendo manualmente a polpa, que foi lavada em água corrente, retirando-se o excesso até a extração das sementes. Posteriormente, as sementes foram 
colocadas para secagem à sombra, por 72 horas à temperatura ambiente.

As avaliações biométricas das sementes foram realizadas seguindo o mesmo procedimento descrito para a biometria dos frutos, onde foram avaliadas as seguintes características:

- Comprimento longitudinal: determinado da base até o ápice da semente;

- Largura: medida na linha mediana central de cada semente;

- Espessura: medida na linha mediana central de cada semente.

Os dados foram submetidos à análise estatística descritiva, classificados por meio de distribuição de frequência e plotados em histogramas de frequência conforme a metodologia recomendada por Oliveira et al. (2000), sendo o número de classes e intervalos de classes determinados de acordo a fórmula de Sturges. Foi calculado o coeficiente de correlação de Spearman (rS) e seu respectivo nível de significância (p-valor) entre as variáveis biométricas através do programa BIOESTAT 5.0 (AYRES et al., 2007).

\section{Resultados e Discussão}

Os resultados da análise estatística descritiva das características biométricas de frutos e sementes de noni estão apresentados na Tabela 1. Foi observado que, a média de comprimento de frutos foi de $89,82 \mathrm{~mm}$ e a média do diâmetro dos frutos foi 46,26 mm. O estudo de Silva et al. (2012), indicou que os frutos de noni quando maduros, apresentaram valor médio do comprimento longitudinal de 101,08 mm e média de diâmetro de 74,41 mm.

Os mesmos autores verificaram ainda que, os frutos de noni podem apresentar tamanhos variáveis de acordo com o estádio de maturação, sendo que, os frutos nos primeiros estádios de maturação são potencialmente menores do que nos estádios subsequentes, porém, quando atingem a maturidade, os frutos reduzem suas dimensões, isso ocorre possivelmente em função do acúmulo de matéria seca e de processos metabólicos durante o processo de maturação e a perda do conteúdo de água para que ocorra acúmulo de matéria seca.

Em relação à assimetria, observou-se que, para comprimento de frutos o valor indicou distribuição assimétrica levemente negativa, enquanto para diâmetro e massa fresca de frutos, os valores corresponderam à 0,84 e 0,70 respectivamente, demonstrando que estas variáveis seguem distribuição assimétrica levemente positiva. 
Tabela1. Análise estatística descritiva para as características biométricas de frutos e sementes de $M$. citrifolia provenientes do município de Boquira, BA.

\begin{tabular}{|c|c|c|c|c|c|c|}
\hline \multirow[t]{2}{*}{ Variável } & Média ( $( \pm \mathrm{EPM})$ & Assimetria & Curtose & $\begin{array}{c}\text { Amplitude } \\
\text { Total }\end{array}$ & $\begin{array}{l}\text { Desvio } \\
\text { Padrão }\end{array}$ & $\mathrm{CV}(\%)$ \\
\hline & \multicolumn{6}{|c|}{ Frutos } \\
\hline Comprimento (mm) & $89,82 \pm 1,85$ & $-0,43$ & 0,65 & 112,38 & 18,52 & 20,62 \\
\hline Diâmetro (mm) & $46,26 \pm 0,58$ & 0,84 & 1,01 & 31,73 & 5,82 & 12,59 \\
\hline \multirow[t]{2}{*}{ Massa fresca (g) } & $113,54 \pm 4,27$ & 0,70 & 0,63 & 243,19 & 42,74 & 37,65 \\
\hline & \multicolumn{6}{|c|}{ Sementes } \\
\hline Comprimento (mm) & $9,96 \pm 0,05$ & $-0,54$ & 1,07 & 3,12 & 0,53 & 5,34 \\
\hline Largura (mm) & $5,91 \pm 0,06$ & 0,59 & 1,47 & 3,59 & 0,58 & 9,78 \\
\hline Espessura (mm) & $2,62 \pm 0,04$ & 0,35 & $-0,37$ & 1,85 & 0,41 & 15,87 \\
\hline
\end{tabular}

Fonte: Própria (2019)

Para a curtose, verificou-se para comprimento, diâmetro e massa fresca os valores de 0,65 1,01 e 0,63 respectivamente, sendo bem próximos de 0 , e isso indica que os dados seguiram uma distribuição leptocúrtica, ou seja, o grau de "achatamento" da curva em função da distribuição dos valores são mais estreitos.

Contudo, os valores de assimetria e curtose encontrados de forma geral, informa que os dados seguem distribuição normal para diâmetro de frutos, enquanto para comprimento e massa fresca ocorre maior dispersão em relação à média. Essa ideia é reforçada pelos valores de amplitude total e desvio padrão encontrados, que para o comprimento foi de 112,38 e 18,52 respectivamente, já para diâmetro dos frutos, a amplitude total foi de 31,73 e o valor de desvio padrão foi de 5,82 .

Os valores obtidos por Silva et al. (2012) para diâmetro de noni em função dos estádios de maturação demonstraram que o fruto de noni quando próximos à maturidade, referidos pelos autores como "de vez", apresentaram maiores variações, resultando em valores médios maiores e maiores coeficientes de variação, porém, quando maduros, os resultados para as mesmas características foram inferiores, no caso deste estudo, a amostra dos frutos selecionada foi composta por frutos obtidos em matrizes desenvolvidas naturalmente na região semiárido, enquanto que a amostra estudada pelos autores no referido estudo foi composta por frutos de pomares comerciais, que receberam manejo adequado de produção.

As variações encontradas para os frutos de noni, podem demonstrar que nas regiões semiáridas, os efeitos dos fatores abióticos influenciam as características biométricas do fruto, e em resposta a estes estímulos, as plantas acabam produzindo frutos de tamanhos diferenciados, e estas diferenças podem ser atribuídas à causas da própria planta como a translocação de 
nutrientes e fotoassimilados de forma irregular entre os frutos e as condições de nutrição, fitossanitárias entre outras.

Em relação as características biométricas da semente, observou-se que a média da amostra para o comprimento foi de $9,96 \mathrm{~mm} ; 5,91 \mathrm{~mm}$ para largura e 2,62 $\mathrm{mm}$ para espessura. Em concordância com o presente trabalho, Paula et al. (2016) encontraram para sementes de noni os valores de comprimento, largura e espessura de 10,07; 5,22 e 2,49 mm, respectivamente.

Os valores de assimetria obtidos para comprimento, largura e espessura das sementes foram de $-0,54 ; 0,59$ e 0,55 respectivamente, já para curtose, os valores para as mesmas variáveis foram de 1,$07 ; 1,47$ e $-0,37$. Quanto a assimetria, apenas para comprimento de sementes foi verificada distribuição assimétrica negativa, a largura e espessura seguiram distribuição assimétrica positiva.

Os valores de desvio padrão foram baixos e os de coeficiente de variação foram inferiores à $20 \%$, indicando que os dados apresentaram pouca variação em relação à media, reforçando que, as características biométricas das sementes apresentaram maior uniformidade, o que não pôde ser verificado para os frutos de noni, que apresentaram grande variação entre os indivíduos das amostras para comprimento e massa fresca, isso possivelmente ocorreu porque as sementes apresentam vantagem quanto aos frutos, pois as mesmas ficam no interior dos frutos, ou que lhes conferem menor exposição aos fatores abióticos, além disso, as plantas de noni produzem alta quantidade de sementes e com tamanhos bem próximos entre si, e menores variações quanto ao tamanho.

Quanto às frequências, a amostra de frutos apresentou valores de comprimento variando entre 29,73 a 128,06 mm (Figura 1A), enquanto para a variável diâmetro, os frutos apresentaram valores variáveis entre 34,94 a 62,60 mm (Figura 1B).

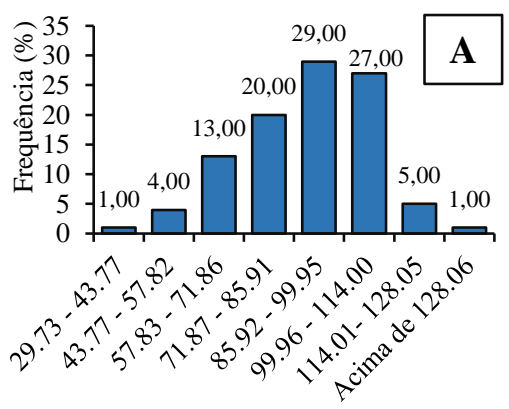

Comprimento (mm)

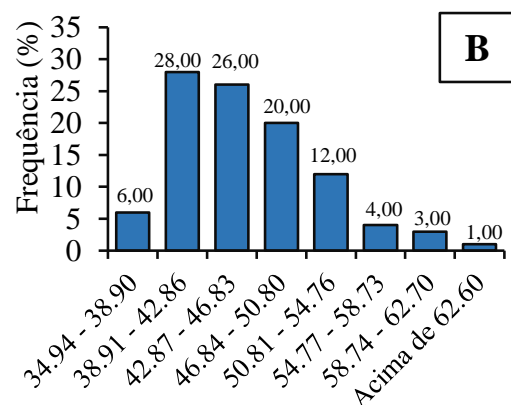

Diâmetro (mm)

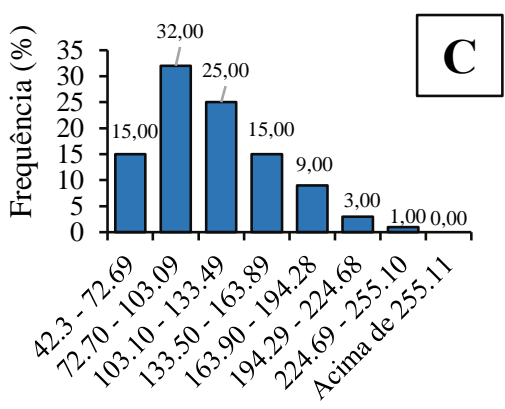

Massa fresca $(\mathrm{g})$

Figura 1. Frequência (\%) de comprimento (mm) (A); Diâmetro (mm) (B) e Massa Fresca (g) (B) de frutos de Morinda citrifolia coletados de diferentes matrizes do município de Boquira-BA. 
Quanto ao comprimento, a maior frequência de frutos (29\%) apresentou valores de 85,92 a 99,95 mm e 99,96 a 114,00 (27\%). Para o diâmetro dos frutos foi encontrado maior porcentagem de frutos variando de 38,91 a 42,86 mm (28\%) e 42,87 a 46,86 mm (26\%).

Avaliando frutos de noni em três estádios de desenvolvimento (verde, de vez e maduro) cultivados no Ceará, Nery et al. (2013), constataram comportamento semelhante ao observado no presente estudo, onde verificaram que os frutos maduros apresentavam cerca de $116,76 \mathrm{~mm}$ de comprimento. Ainda segundo os autores, o diâmetro de frutos encontrado para a amostra foi de 56,66mm, sendo esses valores, superiores aos encontrados nesse trabalho, onde as maiores porcentagem de frutos variaram de 38,91 a 46,83 mm. Segundo Silva et al., (2013) avaliando frutos de noni coletados de cinco matrizes diferentes, a média para o comprimento e diâmetro de frutos foi de 106,60 e 59,96 mm respectivamente. Esses valores indicam que o fruto do noni apresenta formato aproximadamente ovalado. No entanto, as indústrias preferem que os frutos sejam mais arredondados, pois as operações de limpeza, processamento e beneficiamento são executadas com mais facilidade (CHITARRA; CHITARRA, 2005).

Quanto a massa fresca de frutos, observou que os valores variaram entre 42,3 a 255,10 g e cerca de $62 \%$ dos frutos se apresentaram no intervalo entre 72,70 a 103,09 g. Silva et al. (2013), constataram média de 221,50 g e variação entre 195,60 a 258,80 g de massa fresca. Os autores têm ressaltado que a massa do fruto aumenta à medida que o desenvolvimento do fruto tende a maturação, contudo, ocorre decréscimo na massa fresca quando o fruto se atinge estados mais avançados de maturação. Segundo Carvalho e Nakagawa (2000), o aumento gradativo do peso durante o desenvolvimento do fruto ocorre, devido à maior quantidade de fotoassimilados, açúcares e carboidratos acumulados no processo.

Em relação às características biométricas de comprimento, largura e espessura das sementes de noni, verificou-se intervalos de 8,21 a 10,94 mm de comprimento (Figura 2 A); 4,47 a 7,62 mm de largura (Figura 2 B) e 1,81 a 3,44 mm de espessura (Figura 2 C), e a maioria das sementes apresentou comprimento variando entre 9,78 a $10,16 \mathrm{~mm}$, enquanto que a largura se concentrou entre 5,82 a $6,70 \mathrm{~mm}$ e espessura de 2,27 a $2,97 \mathrm{~mm}$. 

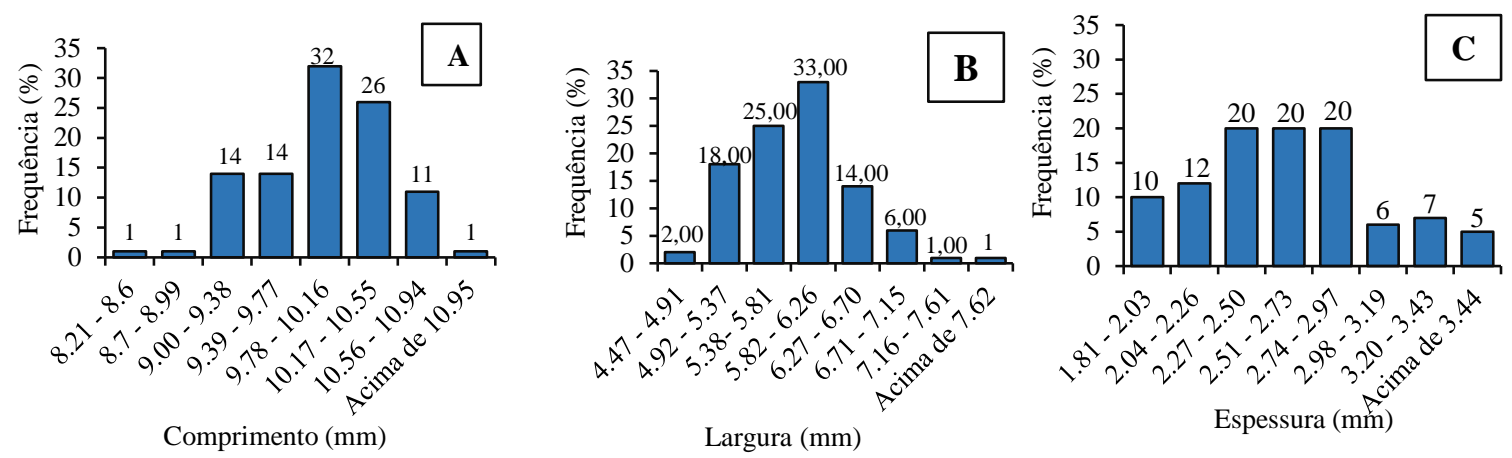

Figura 2. Frequência do comprimento (mm) (A); largura (mm) (B) e espessura (mm) (C) de sementes Morinda citrifolia coletados no município de Boquira-BA.

Conforme Cangussu et al. (2015), em trabalhos com análise biométrica em sementes de noni de diferentes localidades do Estado da Bahia, constatou que o comprimento, largura e espessura variaram de 6,5 a $14,8 \mathrm{~mm}, 2,1$ a $10,4 \mathrm{~mm}$ e 0,3 a $3,8 \mathrm{~mm}$ respectivamente, se assemelhando aos encontrados nesse estudo. Os valores relatados por Paula et al. (2016) com noni, verificou-se comprimento de sementes variável entre 7,96 mm a 12,06 mm, largura entre $3,85 \mathrm{~mm}$ a $7,15 \mathrm{~mm}$ e espessura de $1,57 \mathrm{~mm}$ a $3,36 \mathrm{~mm}$.

O tamanho e o peso das sementes para algumas espécies podem ser considerados indicativo de qualidade fisiológica, sendo que em um mesmo lote, sementes mais leves, normalmente, podem apresentam menor desempenho germinativo do que as sementes mais pesadas, em decorrência da quantidade de reservas acumuladas e da formação do embrião (SANTOS NETO et al., 2009). Segundo Nelson (2005), a dispersão de sementes de noni ocorre sem um agente específico e pode ser realizado por pássaros, morcegos, ratos e outros mamíferos, sendo mais comumente dispersos sobre a água, assim, o tamanho e o peso das sementes assumem importância ecológica para a dispersão das espécies de várias formas, como por exemplo, a barocoria (disseminação da semente pelo peso do fruto), zoocoria (animais), anemocoria (vento), entre outros (DEMINICIS et al., 2009) além de estarem relacionados com à competição, predação e à distribuição espacial da espécie (BRAGA et al., 2007).

A sementes de noni tem a coloração marrom-avermelhada, uma base levemente cônica e topo arredondado, com uma câmara de ar na extremidade oposta à micrópila, que é revestida internamente por uma camada brilhante como celofane (PAULA et al., 2016). A forma de semente noni pode ser classificado como oblongo-triangular, ovoide a obovoide ou reniforme, com uma câmara de ar na extremidade oposta à micrópila e sem asas (MATHIVANAN et al., 
2005; PONNAIYAN; VEZHAVENDAN, 2005; SINGH et al., 2006)

De acordo com Santana et. al (2013) as variações observadas nas dimensões das sementes podem ser atribuídas à variabilidade genética, aos fatores ambientais (bióticos e abióticos), bem como a interação genótipo-ambiente. O estudo da biometria estabelece uma ferramenta eficaz para identificar a variabilidade genética dentro de populações de uma mesma espécie e pode fornecer informações fundamentais para a diferenciação de espécies do mesmo gênero (GUSMÃO et al., 2006).

Os valores de Coeficiente de Spearman (rS), para verificar as associações entre as características biométricas de frutos e sementes e a interferência de uma característica sobre a outra, estão apresentados na Tabela 2. Para o comprimento de frutos, foi verificado correlação significativa e positiva $(\mathrm{p} \leq 0,05)$, porém fraca $(\mathrm{rS}=0,21)$, apenas entre o diâmetro de frutos, onde se observou que com o aumento no comprimento do fruto, o diâmetro do mesmo também aumenta, já para as demais variáveis não houve correlação significativa entre o comprimento dos frutos. As condições edafoclimáticas, genéticas, antrópicas e fenotípicas, bem como a idade das plantas são fatores que influenciam as variações na morfologia dos frutos de determinada espécie, sendo assim, quando ocorrem variações muito altas nos atributos morfológicos, consequentemente maiores correlações entre determinadas características são esperadas.

Tabela 3. Estimativa dos coeficientes de correlação de Spearman (rS) entre os pares das características biométricas de frutos e semente de Morinda citrifolia, Vitória da Conquista, Bahia.

\begin{tabular}{|c|c|c|c|c|c|c|}
\hline & COMPF & DIAMF & MFRSF & COMPS & LARGS & ESPES \\
\hline DIAMF & $0.21 *$ & & & & & \\
\hline MFRSF & $-0.004^{\mathrm{NS}}$ & $0.097^{\mathrm{NS}}$ & & & & \\
\hline COMPS & $-0.09^{\mathrm{NS}}$ & $-0.08^{\mathrm{NS}}$ & $-0.004^{\mathrm{NS}}$ & & & \\
\hline LARGS & $-0.18^{\mathrm{NS}}$ & -0.07 NS & $-0.08^{N S}$ & $0.45 * *$ & & \\
\hline ESPES & $0.04^{\mathrm{NS}}$ & $-0.12^{\mathrm{NS}}$ & $0.17^{\mathrm{NS}}$ & $0.22 *$ & $0.08^{\mathrm{NS}}$ & \\
\hline
\end{tabular}

COMPF- Comprimento do fruto (mm); LARGF- Largura do fruto (mm); ESPEF- Espessura do fruto (mm); MFRSF- Massa fresca do fruto (g); COMPS-Comprimento da semente (mm); LARGS- Largura da semente $(\mathrm{mm})$; ESPES- Espessura da semente $(\mathrm{mm})$

Ns não significativo; ** e * significativo à $1 \%$ e $5 \%$ de probabilidade

Fonte: Própria (2019)

Não houve correlação significativa entre diâmetro dos frutos e nenhuma outra variável avaliada, o mesmo foi verificado para a variável massa fresca dos frutos. Já em relação à variável comprimento de sementes, verificou-se correlação significativa e positiva para largura $(\mathrm{p} \leq 0,01 ; \mathrm{rS}=0,45)$ e espessura $(\mathrm{p} \leq 0,05 ; \mathrm{rS}=0,22)$ da semente, porém fraca nos dois casos, evidenciando que quanto maior for o comprimento da semente, maiores serão os valores de largura e espessura da semente. 
A correlação entre as variáveis biométricas de sementes, contribuem para a seleção indireta de matrizes com variabilidade genética, principalmente nos casos em que as características são de difíceis medições, ou quando as variações são muito baixas entre si, evitando que a tomada de decisão para a seleção de matrizes seja feita de forma empírica. Nesse estudo, os dados apresentaram maio homogeneidade quanto às características biométricas, e, a análise de correlação permitiu identificar qual característica deve ser levada em conta para a seleção de matrizes superiores.

Em trabalhos de biometria de frutos e sementes, é comum ocorrerem associações entre as variáveis de fruto e semente de forma isolada, ou seja, as características biométricas de fruto se correlacionam entre si, mas não apresentam correlação com as variáveis biométricas das sementes e vice-versa, Zuffo et al. (2017) encontraram para frutos de canafístula, correlações positivas e significativas entre largura e comprimento do fruto, entre a massa fresca e massa seca do fruto, já para as sementes de canafístula, os autores verificaram correlação significativa positiva entre comprimento e espessura das sementes, massa fresca e espessura das sementes.

As características biométricas da espécie Morinda citrifolia desse estudo, avaliando apenas os dados da análise estatística descritiva, apresentaram poucas variações entre si, contudo, foi observado que os resultados da correlação demonstraram que existem correlações entre as variáveis biométricas dos frutos e das sementes, sendo assim, o uso da correlação para identificar essas associações podem favorecer a escolha adequada de uma característica para a seleção de matrizes, no caso deste trabalho, as matrizes podem ser selecionadas com base no comprimento dos frutos, se caso o objetivo seja selecionar a matriz que produza frutos maiores, ou com base no comprimento da semente, cujo objetivo da seleção seja a obtenção de sementes maiores, tanto quanto à espessura, quanto à largura.

\section{Conclusões}

Os frutos e sementes de noni proveniente do município de Boquira, apresentaram variabilidade entre as características biométricas avaliadas.

As maiores frequências de comprimento, diâmetro e massa fresca de frutos de noni foram de 85,92 a 99,95 mm, 38,91 a 42,86 $\mathrm{mm}$ e 72,70 a 102,09 g respectivamente.

As frequências de comprimento e largura das sementes foram maiores nos intervalos de 9,78 a $10,16 \mathrm{~mm}$ e 5,82 a $6,26 \mathrm{~mm}$ respectivamente. As sementes de noni apresentaram 
homogeneidade quanto a espessura.

Recomenda-se, a escolha do parâmetro comprimento para frutos e sementes de noni para serem utilizadas na seleção de matrizes.

\section{Referências}

AYRES, M.; AYRES JÚNIOR, M. e SANTOS, A.A.S. BioEstat 5.0: Aplicações estatísticas nas áreas das ciências biológicas e médicas. Belém: Sociedade Civil Mamirauá, 364 p. 2007.

BARBOSA, A. F.; COSTA, I. C. M.; ZUCOLOTTO, S. M.; GIORDANI, R. B. Morinda citrifolia: fatos e riscos sobre o uso do noni. Revista Fitos, Rio de Janeiro, v. 11, n. 2, p. 119249, 2017.

BARROSO, R.F.et al. Biometria de frutos e sementes de Luetzelburgia auriculata (Allemão) Ducke. Revista Verde de Agroecologia e Desenvolvimento Sustentável, v.11, n.5, p.155-160, 2016.

BECHELENI, F. R. C., LEANDRO, O. A. A., JUNIOR, D. C. S., \& DE SOUSA, A. P. G. Importância do consumo de noni (Morinda citrifolia Linn) para a saúde. Revista Brasileira de Ciências da Vida, v. 4, n. 1, 2017.

BERILLI, S. S.; OLIVEIRA, J. G.; MARINHO, A. B.; LYRA, G. B.; SOUSA, E. F.; VIANA, A. P.; BERNARDO, S.; PEREIRA, M. G. Avaliação da taxa de crescimento de frutos de mamão (Carica papaya L.) em função das épocas do ano e graus-dias acumulados. Revista Brasileira de Fruticultura, Jaboticabal, v. 29, n. 1, p. 11-14, 2007.

BRAGA, L. F.; SOUSA, M. P.; GILBERTI, S.; CARVALHO, M. A.C. Caracterização morfométrica de sementes de castanha de sapucaia (Lecythis pisonis Cambess - Lecythidaceae. Revista de Ciências AgroAmbientais, v. 5, n. 1, p. 111-116, 2007.

BRASIL. Ministério da Saúde. Secretaria de Atenção à Saúde. Departamento de Atenção Básica. Política nacional de práticas integrativas e complementares no SUS: atitude de ampliação de acesso / Ministério da Saúde. Secretaria de Atenção à Saúde. Departamento de Atenção Básica. - 2. ed. - Brasília: Ministério da Saúde, 2015.

CANGUSSU, A. C. V.; CARDOSO, A. D.; SANTOS, A. P. S.; BANDEITA, A. S.; MORAIS, O. M. Características morfológicas de sementes de Noni provenientes de frutos de diferentes localidades. In: II simpósio de Agroecologia da Bahia, 2015, Vitória da Conquista. Anais do II Simpósio de Agroecologia da Bahia, 2015.

CANUTO, G. A. B.; XAVIER, A. A, O.; NEVES, L. C.; BENASSI, M. T. Caracterização físico-química de polpas de frutos da Amazônia e sua correlação com a atividade anti-radical livre. Revista Brasileira Fruticultura, Jaboticabal, v. 32, n. 4, p. 1196-1205, 2010.

CARValho, N. M.; NAKAGAWA, J. Sementes: Ciência, Tecnologia e Produção. 5.ed. Jaboticabal: FUNEP, 2012. 590p. 
CHITARRA, M. I. F, CHITARRA, A. B. Pós-colheita de frutos e hortaliças: fisiologia e manuseio. 2. ed. ver. Lavras: UFLA; 2005. p. 785.

COSTA, A. B; OlIVEIRA, A. M. C.; SIlvA, A. M. O; FILHO-MANCINI, J; LIMA, A. Atividade antioxidante da polpa, casca e sementes do noni (Morinda citrifolia Linn). Revista Brasileira de Fruticultura, Jaboticabal - SP, v. 35, n. 2, p. 345-354, 2013.

DA SILVA, L. R., PONTES, C. A., DE SOUSA, J. A., \& DE OLIVEIRA SILVA, E. Calidad de la fruta del noni (Morinda citrifolia L.) cultivado en Trairi-CE. Revista Cubana de Plantas Medicinales, v. 18, n. 1, p. 100-108, 2013.

DEMINICIS, B. B.; VIEIRA, H. D.; ARAÚJO, S. A. C.; JARDIM, J. G.; PÁDUA, F. T.; CHAMBELA NETO, A. Dispersão natural de sementes: importância, classificação e sua dinâmica nas pastagens tropicais. Archivos de Zootecnia, v. 58, p. 35-58, 2009.

FARIA, W. C. S.; BETT, S. C.; SANTOS, C. G. B.; BRASIL, A. S.; GAUTO, R. F.; BESERRA, A. M. S. S.; OLIVEIRA, A. P. Caracterização físico-química e análise fitoquímica preliminar do fruto noni (Morinda citrofolia L.) produzido na cidade de Cuiabá - MT. Revista Brasileira de Tecnologia Agroindustrial, v. 8, n. 1: p. 1208-1215, 2014.

GUSMÃO, E.; VIEIRA, F.A. E FONSECA JÚNIOR, E.M. Biometria de frutos e endocarpos de Murici (Byrsonima verbascifolia Rich. ex A. Juss.). Revista Cerne, v. 12, n. 1, p. 84-91, 2006.

LIMA, C.R , LIMA,R.A et al. Identificação de metabólitos secundários presentes no extrato etanólico dos frutos verdes e maduros de Morinda citrifolia L. Revista Saúde e Pesquisa, v. 6, n. 3, p. 439-446, set./dez. 2013.

MATHIVANAN, N.; SURENDIRAN, G.; SRINIVASAN, K.; SAGADEVAN, E.; MALARVIZHI, K. Review on the current scenario of Noni research: taxonomy, distribution, chemistry, medicinal and therapeutic values of Morinda citrifolia. International Journal Noni Research, v. 1, p. 1-16, 2005.

MATOSO, LEONARDO MAGELA LOPES et al. As Características e a Utilização do Noni (Morinda Citrifolia). Ciência \& Desenvolvimento-Revista Eletrônica da FAINOR, v. 6, n. 1, p. 42- 50, jan/jun. 2013.

NASCIMENTO, A, L. C. et al. Atividade antioxidante do extrato aquoso de Noni em diluente para congelação. Boletim de Indústria Animal, v.73, n.1, p.68-74, 2016.

NERY, K. A.; ARAUJO, R. O.; BRAGA, T. R.; OLIVEIRA, M. M. T.; TORRES, L. B. V.; SILVA, L. R. Caracterização física e físico-química de frutos do noni (Morinda citrifolia L.) cultivados em Fortaleza-CE. Revista Cultivando o Saber, Cascavel-PR, v.6, p.17-24, 2013.

OLIVEIRA, A.N.; QUEIROZ, M.S.M.; RAMOS, M.B.P. Estudo morfológico de frutos e sementes de trefósia (Tephrosia candida DC.- Papiloinoideae) na Amazônia Central. Revista Brasileira de Sementes, v.22, n.2, p.193-199, 2000. 
PAULA, S. D. O.; SOUSA, J. A. D.; BRITO, E. S. D.; GALLÃO, M. I. The morphological characterization of the dry seeds and reserve mobilization during germination in Morinda citrifolia L. Revista Ciência Agronômica, v. 47, n. 3, p. 556-563, 2016.

PAULA, S. de O.; SOUSA, J.A.; BRITO, E.S. de; GALLÃO, M.I. The morphological characterization of the dry seeds and reserve mobilization during germination in Morinda citrifolia L. Revista Ciência Agronômica, v. 47, n. 3, p. 556-563, 2016.

PONNAIYAN, P.; VEZHAVENDAN, S. The effect of hot water and acid sulphuric acido on the seed germination of Morinda citrifolia L. International Journal Noni Research, v. 1, p. 36$39,2005$.

SANTANA, S.H.; TORRE, S.B.; BENEDITO, C.P. Biometria de frutos e sementes e germinação de Melão-de-São-Caetano. Revista Brasileira Plantas Medicinais, v.15, n. 2, Botucatu 2013.

SANTOS NETO, A. L.; MEDEIROS FILHO, S.; BLANK, A. F.; SANTOS, V. R.; ARAÚJO, E. Influência do peso da semente e promotores químicos na qualidade fisiológica de sementes de sambacaitá. Revista Caatinga, Mossoró, v.22, n.1, p.187-192, 2009.

SEI - SUPERINTENDÊNCIA DE ESTUDOS ECONÔMICOS E SOCIAIS DA BAHIA. Estatística dos Municípios Baianos. Salvador - BA, v. 19; 182 p., 2011.

SILVA, C.L.R. da; MEDEIROS, P.V.Q. de; LEITE, G.A.; SILVA, K.J.P. MENDONÇA, V. SILVA, G.G.da. Caracterização do fruto de Morinda citrifolia L. (noni). Revista Cubana de Plantas Medicinales, v. 17, n. 1, p. 93-100, 2012.

SINGH, D. R. Seed germination studies on Morinda citrifolia. International Journal Noni Research, v. 1, p. 23-28, 2006.

ZUFFO, A. M.; STEINER, f.; ZOZ, T.; ZUFFO JÚNIOR, J.M; DOURADINHO, G.Z.; OLIVEIRA, C.P.; BORTOLAZZO, G. Atributos biométricos de frutos e sementes de Peltophorum dubium (Spreng.) Taub. Revista de Ciências Agrárias, Lisboa, v. 40, n. 1, p. 6168, 2017. 